УДК 514.157.2

\title{
The Trace Theorem for Anisotropic Sobolev - Slobodetskii Spaces with Applications to Nonhomogeneous Elliptic BVPs
}

S.A. Sazhenkov ${ }^{1,2,3}$ and E.V. Sazhenkova ${ }^{4}$

${ }^{1}$ Lavrentiev Institute of Hydrodynamics of the Siberian Branch of the RAS

(Novosibirsk, Russia)

${ }^{2}$ Novosibirsk State University (Novosibirsk, Russia)

${ }^{3}$ Heilongjiang University, Sino-Russian Institute (Harbin, PR China)

${ }^{4}$ Novosibirsk State University of Economics and Management (Novosibirsk, Russia)

\section{Теорема о следах в анизотропных пространствах Соболева - Слободецкого и ее приложения к неоднородным краевым эллиптическим задачам}

\author{
C.A. Саженков ${ }^{1,2,3}$, Е.В. Саженкова ${ }^{4}$ \\ ${ }^{1}$ Институт гидродинамики им. М.А. Лаврентьева СО РАН (Новосибирск, Россия) \\ ${ }^{2}$ Новосибирский государственный университет (Новосибирск, Россия) \\ ${ }^{3}$ Хэйлунцзянский университет, КРИ (Харбин, Китай) \\ ${ }^{4}$ Новосибирский государственный университет экономики и управления \\ (Новосибирск, Россия)
}

In this paper, anisotropic Sobolev - Slobodetskii spaces in poly-cylindrical domains of any dimension $N$ are considered. In the first part of the paper we revisit the well-known Lions - Magenes Trace Theorem (1961) and, naturally, extend regularity results for the trace and lift operators onto the anisotropic case. As a byproduct, we build a generalization of the Kruzhkov - Korolev Trace Theorem for the first-order Sobolev Spaces (1985). In the second part of the paper we observe the nonhomogeneous Dirichlet, Neumann, and Robin problems for p-elliptic equations. The well-posedness theory for these problems can be successfully constructed using isotropic theory, and the corresponding results are outlined in the paper. Clearly, in such a unilateral approach, the anisotropic features are ignored and the results are far beyond the critical regularity. In the paper, the refinement of the trace theorem is done by the constructed extension. Namely, we formulate proper weakly regular anisotropic classes for boundary conditions, so that the boundary value problems appear to be well-posed. Finally, the analogous results are formulated for the $p$-parabolic problems.

Key words: anisotropic fractional Sobolev spaces, boundary trace of function, p-Laplace operator.

DOI 10.14258/izvasu(2018)4-19

The first author's work was supported by the Ministry of Higher Education and Science of the Russian Federation (project no. III.22.4.2) and by the Russian Foundation For Basic Research (grant code 18-01-00649).
Рассматриваются анизотропные пространства Соболева - Слободецкого в ограниченных полицилиндрических областях произвольной размерности $N$. В первой части статьи конструируется естественное продолжение результатов теоремы Лионса - Мадженеса о граничных следах функций, принадлежащих изотропным пространствам (1961), на случай анизотропных пространств. Как следствие, также устанавливается некоторое обобщение теоремы Кружкова - Королёва о граничных следах функций (1985) для соболевских пространств первого порядка. Во второй части статьи рассматриваются основные неоднородные краевые задачи для вырождающихся эллиптических уравнений с анизотропным р-лапласианом. Результаты о корректности этих задач, получаемые с помощью результатов и техники Лионса - Мадженеса, разработанной для изотропных пространств, корректны, но не учитывают особенностей, связанных с анизотропией. В связи с этим проводится уточнение: с помощью построенного в первой части статьи продолжения теории следов формулируются надлежащие слабо регулярные анизотропные классы для граничных условий, для которых рассматриваемые задачи оказываются корректно поставленными.

Ключевые слова: анизотропное пространство Соболева дробного порядка, р-лапласиан, граничный след функции.

Introduction This article is devoted to a study of a class of anisotropic Sobolev - Slobodetskii spaces $W^{s, p}(\mathcal{O})$ and their dual spaces $W^{-s, p^{\prime}}(\mathcal{O})$. Here domain $\mathcal{O}$ is a polycylinder in $\mathbb{R}_{x}^{N}$, i.e., it meets the following requirements. 
Condition C. $\bullet \mathcal{O}=\mathcal{B}_{1} \times \mathcal{B}_{2} \times \ldots \times \mathcal{B}_{k}$,

- $\mathcal{B}_{i} \subset \mathbb{R}_{\boldsymbol{x}_{i}}^{N_{i}}(i=1,2, \ldots, k), \quad \sum_{i=1}^{k} N_{i}=N$

- $\mathcal{B}_{i}$ are nonempty bounded open sets with Lipschitz boundaries $\partial \mathcal{B}_{i}$.

Multi-indices $\boldsymbol{s}=\left(s_{1}, s_{2}, \ldots, s_{k}\right)$ and $\boldsymbol{p}=$ $\left(p_{1}, p_{2}, \ldots, p_{k}\right)$ are such that $s_{i} \in(0,1]$ and $p_{i} \in(1,+\infty)$. Thus $W^{\boldsymbol{s}, \boldsymbol{p}}(\mathcal{O})$ are fractional Sobolev spaces, in general. Multi-index $\boldsymbol{p}^{\prime}=\left(p_{1}^{\prime}, p_{2}^{\prime}, \ldots, p_{k}^{\prime}\right)$ is such that $p_{i}^{-1}+\left(p_{i}^{\prime}\right)^{-1}=1(i=1,2, \ldots, k)$, thereby $p_{i}^{\prime} \in(1,+\infty)$. We focus on the question about regularity properties of traces of functions from $W^{\boldsymbol{s}, \boldsymbol{p}}(\mathcal{O})$ on subsets of $\partial \mathcal{O}$. Our interest to this question is motivated by applications to $\boldsymbol{p}$-elliptic equations (see Eq. (7) in Section 2.) supplemented by either Dirichlet or Neumann nonhomogeneous conditions. Such equations arise in modelling of heat transfer, gas diffusion, etc [1-6]. Anisotropy $p_{i} \neq p_{j}$ means that diffusion rates differ in different directions $\boldsymbol{x}_{i}$ and $\boldsymbol{x}_{j}$.

The general theory of isotropic Sobolev Slobodetskii spaces was built in sixtieth of 20th century [7-9]. Within its framework, regularity properties of traces of functions $\phi: \mathbb{R}_{x}^{N} \mapsto \mathbb{R}$ on $(N-$ $1)$-dimensional manifolds were investigated in detail (see, for example, [7, Theorem 5.1], [10, Theorems 2.21 and 2.22]). Some applications of trace theorems to the Dirichlet, Neumann, and Robin problems for the isotropic $p$-Laplacian equation can be found in [11, Chapter 2]. Embedding in anisotropic Sobolev spaces of the first order were studied in [12], and the following result was established [12, Inequality (12)] (see also [1, Lemma 3.6 and Remark 3.6]):

Proposition 1. Let $\Omega \subset \mathbb{R}_{\boldsymbol{x}}^{N}$ be a bounded domain with the smooth boundary $\Gamma, u \in L^{r}(\Omega), \partial_{x_{i}} u \in$ $L^{p_{i}}(\Omega), 1<p_{i}<\infty, i=1, \ldots, N$.

Then

$$
\begin{aligned}
& \|u\|_{L^{q}(\Gamma)} \leq \\
\leq & C(\Omega)\left(\sum_{k=1}^{N}\left|\partial_{x_{i}} u\right|_{L^{p_{i}(\Omega)}}+\|u\|_{L^{r}(\Omega)}\right)^{\theta}\|u\|_{L^{r}(\Omega)}^{1-\theta},
\end{aligned}
$$

where

$$
\begin{gathered}
\frac{N}{p^{*}} \leq q, 1<r \leq q, p^{*}=\sum_{i=1}^{N} \frac{1}{p_{i}} \\
\frac{1}{N\left(1-p^{*}\right)}+\frac{1}{r}>0, \\
\theta=\left(\frac{1}{r}-\frac{N-1}{N q}\right) /\left(\frac{1}{N}-\frac{1}{N p^{*}}+\frac{1}{r}\right) .
\end{gathered}
$$

Also, worth to notice the results on traces in anisotropic spaces that were achieved in $[13,14]$.

The present article is organized as follows.
In Section 1, we formulate the trace theorem for anisotropic Sobolev - Slobodetskii spaces (Theorem 1). Then we outline a brief scheme of its proof. This theorem somewhat extends the result of Proposition 1 , in particular.

In Section 2, we give some examples of application of this theorem to the basic nonhomogeneous boundary value problems.

1. The Trace Theorem in Anisotropic Sobolev - Slobodetskii Spaces

1.1. Some useful notation. In Introduction we have entered a polycylindrical domain $\mathcal{O} \subset \mathbb{R}_{\boldsymbol{x}}^{N}$ satisfying Condition C. Now let us introduce some relevant convenient notation in order to study traces of functions from $W^{\boldsymbol{s}, \boldsymbol{p}}(\mathcal{O})$ on $(N-1)$-dimensional manifolds $\mathcal{M} \subset \partial \mathcal{O}$. For $\boldsymbol{x} \in \mathcal{O}$ write

$$
\boldsymbol{x}=\left(\boldsymbol{x}_{1}, \boldsymbol{x}_{2}, \ldots, \boldsymbol{x}_{k}\right) \in \mathcal{O},
$$

where $\boldsymbol{x}_{i} \in \mathcal{B}_{i}(i=1, \ldots, k)$, and denote

$$
\begin{aligned}
& \widehat{\boldsymbol{x}}_{1}:=\left(\boldsymbol{x}_{2}, \boldsymbol{x}_{3}, \ldots, \boldsymbol{x}_{k}\right) \in \mathcal{O}_{\widehat{x}_{1}}, \\
& \mathcal{O}_{\widehat{x}_{1}}:=\mathcal{B}_{2} \times \mathcal{B}_{3} \times \ldots \times \mathcal{B}_{k} \subset \mathbb{R}^{N-N_{1}} \text {, } \\
& \vdots \\
& \widehat{\boldsymbol{x}}_{j}:=\left(\boldsymbol{x}_{1}, \ldots, \boldsymbol{x}_{j-1}, \boldsymbol{x}_{j+1}, \ldots, \boldsymbol{x}_{k}\right) \in \mathcal{O}_{\widehat{x}_{j}}, \\
& \mathcal{O}_{\widehat{x}_{j}}:=\mathcal{B}_{1} \times \ldots \times \mathcal{B}_{j-1} \times \mathcal{B}_{j+1} \times \ldots \times \mathcal{B}_{k} \subset \\
& \subset \mathbb{R}^{N-N_{j}} \quad(j=2,3, \ldots, k-1), \\
& \vdots \\
& \widehat{\boldsymbol{x}}_{k}:=\left(\boldsymbol{x}_{1}, \ldots, \boldsymbol{x}_{k-2}, \boldsymbol{x}_{k-1}\right) \in \mathcal{O}_{\widehat{x}_{k}}, \\
& \mathcal{O}_{\widehat{x}_{k}}:=\mathcal{B}_{1} \times \ldots \times \mathcal{B}_{k-2} \times \mathcal{B}_{k-1} \subset \mathbb{R}^{N-N_{k}} \text {. }
\end{aligned}
$$

For the sake of conciseness, for $\phi: \overline{\mathcal{O}} \mapsto \mathbb{R}$ we write

$$
\begin{aligned}
& \phi\left(\widehat{\boldsymbol{x}}_{1} ; \boldsymbol{\zeta}_{1}\right):=\phi\left(\boldsymbol{\zeta}_{1}, \boldsymbol{x}_{2}, \boldsymbol{x}_{3}, \ldots, \boldsymbol{x}_{k}\right) \quad\left(\boldsymbol{\zeta}_{1} \in \overline{\mathcal{B}}_{1}\right), \\
& \vdots \\
& \phi\left(\widehat{\boldsymbol{x}}_{j} ; \boldsymbol{\zeta}_{j}\right):=\phi\left(\boldsymbol{x}_{1}, \ldots, \boldsymbol{x}_{j-1}, \boldsymbol{\zeta}_{j}, \boldsymbol{x}_{j+1}, \ldots, \boldsymbol{x}_{k}\right) \\
& \quad\left(\boldsymbol{\zeta}_{j} \in \overline{\mathcal{B}}_{j}, j=2,3, \ldots, k-1\right), \\
& \vdots \\
& \phi\left(\widehat{\boldsymbol{x}}_{k} ; \boldsymbol{\zeta}_{k}\right):=\phi\left(\boldsymbol{x}_{1}, \ldots, \boldsymbol{x}_{k-1}, \boldsymbol{\zeta}_{k}\right) \quad\left(\boldsymbol{\zeta}_{k} \in \overline{\mathcal{B}}_{k}\right),
\end{aligned}
$$

when it is suitable.

Quite analogously, we also introduce further the notation $\widehat{\boldsymbol{s}}_{i}$ and $\widehat{\boldsymbol{p}}_{i}$.

Remark 1. In line with the above introduced notation, remark that $\partial \mathcal{O}=\bigcup_{i=1}^{k} \partial \mathcal{B}_{i} \times \overline{\mathcal{O}}_{\widehat{x}_{i}}$.

Of course, in the right-hand side here we mean that the order of Cartesian product is proper, that is, by $\partial \mathcal{B}_{i} \times \overline{\mathcal{O}}_{\widehat{x}_{i}}$ we mean

$$
\overline{\mathcal{B}}_{1} \times \ldots \times \overline{\mathcal{B}}_{i-1} \times \partial \mathcal{B}_{i} \times \overline{\mathcal{B}}_{i+1} \times \ldots \times \overline{\mathcal{B}}_{k} .
$$

The above introduced notation will be systematically used throughout the rest of the article. 
1.2. Notion of anisotropic Sobolev Slobodetskii spaces For $\boldsymbol{p}=\left(p_{1}, p_{2}, \ldots, p_{k}\right)$ introduce the anisotropic Lebesgue space $L^{p}(\mathcal{O})$ equipped with the standard norm

$$
\|\phi\|_{L} \boldsymbol{p}(\mathcal{O})=\|\ldots\|\|\phi\|_{L^{p_{1}}\left(\mathcal{B}_{1}\right)}\left\|_{L^{p_{2}\left(\mathcal{B}_{2}\right)}} \cdots\right\|_{L^{p_{k}\left(\mathcal{B}_{k}\right)}},
$$

where, as usually,

$$
\left\|\Psi\left(\widehat{\boldsymbol{x}}_{i} ; \cdot\right)\right\|_{L^{p_{i}\left(\mathcal{B}_{i}\right)}}=\left(\int_{\mathcal{B}_{i}}\left|\Psi\left(\widehat{\boldsymbol{x}}_{i} ; \boldsymbol{\zeta}_{i}\right)\right|^{p_{i}} d \boldsymbol{\zeta}_{i}\right)^{1 / p_{i}}
$$

for sufficiently regular $\Psi$.

Further, for $s_{i} \in(0,1)$ and sufficiently smooth $\phi$ : $\overline{\mathcal{O}} \mapsto \mathbb{R}$ introduce the seminorm

$$
\begin{aligned}
& \langle\phi\rangle_{W_{x_{i}}^{s_{i}, p_{i}}(\mathcal{O})}^{p^{O_{2}}}= \\
= & \int_{\mathcal{O}_{\widehat{x}_{i}}}\left[\int_{\mathcal{B}_{i}} \int_{\mathcal{B}_{i}} \frac{\left|\phi\left(\widehat{\boldsymbol{x}}_{i} ; \boldsymbol{\zeta}_{i}\right)-\phi\left(\widehat{\boldsymbol{x}}_{i} ; \boldsymbol{\eta}_{i}\right)\right|^{p_{i}}}{\left|\boldsymbol{\zeta}_{i}-\boldsymbol{\eta}_{i}\right|^{N_{i}+p_{i} s_{i}}} d \boldsymbol{\zeta}_{i} d \boldsymbol{\eta}_{i}\right] d \widehat{\boldsymbol{x}}_{i} .
\end{aligned}
$$

For $s_{i}=1$ we canonically define

$$
\langle\phi\rangle_{W_{x_{i}}^{1, p_{i}}(\mathcal{O})}=\left\|\nabla_{x_{i}} \phi\right\|_{L^{p_{i}(\mathcal{O})}} .
$$

Now we are in a position to define anisotropic Sobolev - Slobodetskii spaces $W^{\boldsymbol{s}, \boldsymbol{p}}(\mathcal{O})$ in a rigorous way.

Definition 1. The space of functions $\phi: \mathcal{O} \mapsto \mathbb{R}$ equipped with the Sobolev - Slobodetskii norm

$$
\|\phi\|_{W^{\boldsymbol{s}, \boldsymbol{p}(\mathcal{O})}}=\|\phi\|_{L} \boldsymbol{p}_{(\mathcal{O})}+\sum_{i=1}^{k}\langle\phi\rangle_{W_{x_{i}}^{s_{i}, p_{i}}(\mathcal{O})}
$$

is called the Sobolev - Slobodetskii space $W^{\boldsymbol{s}, \boldsymbol{p}}(\mathcal{O})$.

In order to study properties of traces of $\phi \in$ $W^{\boldsymbol{s}, \boldsymbol{p}}(\mathcal{O})$ on $(N-1)$-dimensional manifolds $\mathcal{M} \subset \partial \mathcal{O}$, we also introduce the notion of $W^{\gamma, r}(\mathcal{M})$. To this end, we naturally induce the norm of $W^{\boldsymbol{s}, \boldsymbol{p}}(\mathcal{O})$ onto any $(N-1)$-dimensional Lipschitz manifold $\mathcal{M} \subset \partial \mathcal{O}$ via its atlas, and set

$$
\|\phi\|_{W}^{\boldsymbol{\gamma}, \boldsymbol{r}(\mathcal{M})}:=\|\phi\|_{L^{\boldsymbol{r}}(\mathcal{M})}+\sum_{i=1}^{k}\langle\phi\rangle_{W_{x_{i}}^{\gamma_{i}, r_{i}}\left(\mathcal{M} \cap \partial \mathcal{B}_{i}\right)},
$$

where

$$
\begin{aligned}
& \|\phi\|_{L^{\boldsymbol{r}}(\mathcal{M})}= \\
& \quad=\sum_{i=1}^{k}\left(\int_{\mathcal{M} \cap \partial \mathcal{B}_{i}}\left\|\phi\left(\cdot ; \boldsymbol{x}_{i}\right)\right\|_{L^{\boldsymbol{r}_{i}\left(\mathcal{O}_{\widehat{x}_{i}}\right)}}^{r_{i}} d \boldsymbol{\sigma}_{x_{i}}\right)^{1 / r_{i}}, \\
& \langle\phi\rangle_{W_{x_{i}}^{r_{i}}}^{\gamma_{i}, r_{i}}\left(\mathcal{M} \cap \partial \mathcal{B}_{i}\right) \\
& \quad=\int_{\mathcal{O}_{\widehat{x}_{i}}} \int_{\mathcal{M} \cap \partial \mathcal{B}_{i}} \int_{\mathcal{M} \cap \partial \mathcal{B}_{i}} \mathcal{A}\left(\widehat{\boldsymbol{x}}_{i}, \boldsymbol{\eta}_{i}, \boldsymbol{\zeta}_{i}\right) d \boldsymbol{\sigma}_{\zeta_{i}} d \boldsymbol{\sigma}_{\eta_{i}} d \widehat{\boldsymbol{x}}_{i},
\end{aligned}
$$$$
\mathcal{A}\left(\widehat{\boldsymbol{x}}_{i}, \boldsymbol{\eta}_{i}, \boldsymbol{\zeta}_{i}\right):=\frac{\left|\phi\left(\widehat{\boldsymbol{x}}_{i} ; \boldsymbol{\zeta}_{i}\right)-\phi\left(\widehat{\boldsymbol{x}}_{i} ; \boldsymbol{\eta}_{i}\right)\right|^{r_{i}}}{\left|\boldsymbol{\zeta}_{i}-\boldsymbol{\eta}_{i}\right|^{N_{i}-1+r_{i} \gamma_{i}}},
$$

$\gamma_{i} \in(0,1), r_{i} \in(1,+\infty) ;$ and $d \boldsymbol{\sigma}_{x_{i}}, d \boldsymbol{\sigma}_{\zeta_{i}}$, and $d \boldsymbol{\sigma}_{\eta_{i}}$ are elements of $\mathcal{M} \cap \partial \mathcal{B}_{i}$.

Definition 2. The space of functions $\phi: \mathcal{M} \mapsto \mathbb{R}$ equipped with the norm (1) is called the SobolevSlobodetskii space $W^{\boldsymbol{\gamma}, \boldsymbol{r}}(\mathcal{M}), \mathcal{M} \subset \partial \mathcal{O}$.

Remark 2. The important case is $\mathcal{M}=\partial \mathcal{O}$. Obviously, restriction of $\phi \in W^{\boldsymbol{\gamma}, \boldsymbol{r}}(\partial \mathcal{O})$ to $\mathcal{M} \subset \partial \mathcal{O}$ $\left(\mathcal{M}\right.$ Lipschitz) belongs to $W^{\boldsymbol{\gamma}, \boldsymbol{r}}(\mathcal{M})$. If $\mathcal{M} \cap \partial \mathcal{B}_{i}=\emptyset$ then $\gamma_{i}$ and $r_{i}$ are dumb indices and therefore can be taken arbitrarily.

Remark 3. If $p_{1}=\ldots=p_{k}=p \in(1,+\infty)$ and $s_{1}=\ldots=s_{k}=s \in(0,1]$ then $W^{s, p}(\mathcal{O}):=W^{\boldsymbol{s}, \boldsymbol{p}}(\mathcal{O})$ is the classical isotropic Soboles $(s=1)$ or Sobolev Slobodetskii $(s \in(0,1))$ space. If $s_{1}=\ldots=s_{k}=1$ and $p_{i} \neq p_{j}$ for $i \neq j$, in general, then $W^{1, p}(\mathcal{O})$ is the anisotropic first-order Sobolev space, as in Proposition 1.

We finish this subsection by introducing the notion of the negative spaces.

Definition 3. For $\gamma_{i} \in(0,1)$ and $r_{i} \in(1,+\infty)(i=$ $1, \ldots, k)$, for an arbitrarily fixed $(N-1)$-dimensional Lipschitz manifold $\mathcal{M} \subset \partial \mathcal{O}$, the space $W^{-\boldsymbol{\gamma}, \boldsymbol{r}^{\prime}}(\mathcal{M})$ is defined as the dual space of $W^{\boldsymbol{\gamma}, \boldsymbol{r}}(\mathcal{M})$. Hereby we have $1 / r_{i}+1 / r_{i}^{\prime}=1$, and the associated norm is

$$
\|\Lambda\|_{W^{-\boldsymbol{\gamma}, \boldsymbol{r}^{\prime}}(\mathcal{M})}=\sup _{\substack{W \boldsymbol{\gamma}, \boldsymbol{r}_{(\mathcal{M})} \\ \phi \neq 0}} \frac{\left|\langle\Lambda, \phi\rangle_{\mathcal{M}}\right|}{\|\phi\|_{W} \boldsymbol{\gamma}, \boldsymbol{r}(\mathcal{M})} .
$$

Analogously, $W^{-1, \boldsymbol{r}^{\prime}}(\mathcal{O})=\left(W^{1, \boldsymbol{r}}(\mathcal{O})\right)^{*}$.

By $\langle\cdot, \cdot\rangle_{\mathcal{M}}$ we denote the duality bracket between $W^{\boldsymbol{\gamma}, \boldsymbol{r}}(\mathcal{M})$ and $W^{-\boldsymbol{\gamma}, \boldsymbol{r}^{\prime}}(\mathcal{M})$. Definition 3 generalizes the notion of negative Sobolev - Slobodetskii spaces from isotropic case [7, Section 4.3], [10, Section 2.2] onto anisotropic case.

1.3. Notion of interior trace. Formulation of the trace theorem in anisotropic spaces.

Definition 4. For $\phi \in \mathcal{D}(\overline{\mathcal{O}})$ the mapping $\gamma_{0}^{\text {int }}$ defined by the formula

$$
\gamma_{0}^{i n t} \phi(\boldsymbol{x}):=\lim _{\substack{\tilde{\boldsymbol{x}} \rightarrow \boldsymbol{x} \\ \tilde{\boldsymbol{x}} \in \mathcal{O} \\ \boldsymbol{x} \in \partial \mathcal{O}}} \phi(\tilde{\boldsymbol{x}}) \quad \text { for } \boldsymbol{x} \in \partial \mathcal{O}
$$

is called the interior boundary trace operator.

Here $\mathcal{D}(\overline{\mathcal{O}})$ is the space of $\mathcal{C}^{\infty}$ functions with compact support contained in $\overline{\mathcal{O}}$.

The following theorem is the first main result of the article. 
Theorem 1. (The Trace Theorem in Anisotropic Spaces.) Let $\mathcal{O} \subset \mathbb{R}_{\boldsymbol{x}}^{N}$ be a polycylinder that meets the requirements of Condition $C$. Then the following assertions hold true.

(i) For any $\boldsymbol{p}=\left(p_{1}, p_{2}, \ldots, p_{k}\right), p_{i} \in(1,+\infty)$, the interior boundary trace operator $\gamma_{0}^{\text {int }}$, defined by (2) for $\phi \in \mathcal{D}(\mathcal{O})$, admits a continuous extension

$$
\begin{gathered}
\gamma_{0}^{i n t} \in \mathcal{L}\left(W^{1, \boldsymbol{p}}(\mathcal{O}), W^{\boldsymbol{\gamma}, \boldsymbol{p}}(\partial \mathcal{O})\right), \\
\gamma=\left(\frac{1}{p_{1}^{\prime}}, \frac{1}{p_{2}^{\prime}}, \ldots, \frac{1}{p_{k}^{\prime}}\right), \quad \frac{1}{p_{i}}+\frac{1}{p_{i}^{\prime}}=1,
\end{gathered}
$$

hereby there is a constant $c_{T}>0$ such that

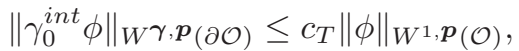

$$
\begin{aligned}
& \forall \phi \in W^{1, p}(\mathcal{O}) .
\end{aligned}
$$

(Constant $c_{T}$ is independent of $\left.\phi.\right)$

(ii) For any $\boldsymbol{p}=\left(p_{1}, p_{2}, \ldots, p_{k}\right) p_{i} \in(1,+\infty)$, the interior boundary trace operator $\gamma_{0}^{\text {int }}$ has a continuous right inverse operator (called the lift operator)

$$
\mathcal{E} \in \mathcal{L}\left(W^{\boldsymbol{\gamma}, \boldsymbol{p}}(\partial \mathcal{O}), W^{1, \boldsymbol{p}}(\mathcal{O})\right)
$$

satisfying $\gamma_{0}^{\text {int }} \mathcal{E} \psi=\psi$ for all $\psi \in W^{\boldsymbol{\gamma}, \boldsymbol{p}}(\partial \mathcal{O})$ as well as

$$
\begin{aligned}
&\|\mathcal{E} \psi\|_{W^{1,}, \boldsymbol{p}_{(\mathcal{O})} \leq c_{I T}\|\psi\|_{W} \boldsymbol{\gamma}, \boldsymbol{p}_{(\partial \mathcal{O})},} \\
& \forall \psi \in W^{\boldsymbol{\gamma}, \boldsymbol{p}}(\partial \mathcal{O}) .
\end{aligned}
$$

(Constant $c_{I T}$ is independent of $\psi$. Notation $\gamma$ is the same as in (4).)

Remark 4. Let $\mathcal{M} \subset \partial \mathcal{O}$ be a $(N-1)$-dimensional Lipschitz manifold. On the strength of Remark 2, the both assertions of Theorem 1 hold true with $\mathcal{M}$ on the place of $\partial \mathcal{O}$.

1.4. Brief scheme of proof of Theorem $\mathbf{1 .}$ The idea of the proof is very simple. Firstly, we directly apply the Lions - Magenes Trace Theorem [7, Theorem 5.1] (in the isotropic case) to the space of functions $\left(\boldsymbol{x}_{i} \mapsto \phi\left(\widehat{\boldsymbol{x}}_{i} ; x_{i}\right)\right) \in W^{1, p_{i}}\left(\mathcal{B}_{i}\right)$ for a.e. $\widehat{\boldsymbol{x}}_{i} \in \overline{\mathcal{O}}_{\widehat{x}_{i}}$ and construct the trace operators

$$
\begin{gathered}
\gamma_{0}^{\text {int }, \mathcal{B}_{i}}: \mathcal{B}_{i} \mapsto \partial \mathcal{B}_{i}, \\
\gamma_{0}^{i n t, \mathcal{B}_{i}} \in \mathcal{L}\left(W^{1, p_{i}}\left(\mathcal{B}_{i}\right), W^{1 / p_{i}^{\prime}, p_{i}}\left(\partial \mathcal{B}_{i}\right)\right)
\end{gathered}
$$

$(i=1,2, \ldots, k)$. These operators depend on $\widehat{\boldsymbol{x}}_{i} \in \overline{\mathcal{O}}_{\widehat{x}_{i}}$ parametrically.

Secondly, we notice that, since $\mathcal{O}$ is polycylinder, then variables $\boldsymbol{x}_{1}, \boldsymbol{x}_{2}, \ldots, \boldsymbol{x}_{k}$ are separated, so that the operator $\gamma_{0}^{\text {int }}$ defined by the rule

$$
\begin{array}{r}
\gamma_{0}^{i n t} \phi(\boldsymbol{x})=\gamma_{0}^{i n t, \mathcal{B}_{i}} \phi\left(\widehat{\boldsymbol{x}}_{i} ; \boldsymbol{x}_{i}\right) \text { for } \boldsymbol{x} \in \partial \mathcal{B}_{i} \\
(i=1,2, \ldots, k)
\end{array}
$$

satisfies (3) and is the trace operator by construction.

Thirdly, thus constructed $\gamma_{0}^{\text {int }}$ is surjective since all $\gamma_{0}^{\text {int }, \mathcal{B}_{i}}$ are surjective and the sets $\partial \mathcal{B}_{i} \times \overline{\mathcal{O}}_{\widehat{x}_{i}}$ do not overlap each other. Hence the right inverse operator $\mathcal{E}$ is also defined and satisfies (5).
2. On Boundary Value Problems for the Anisotropic $p$-Laplacian Equations. In this section we revisit the theory of weak generalized solutions to the Dirichlet, Neumann, and Robin problems for the isotropic $p$-Laplacian equation. This theory was mainly built in [11, Chapter 2]. With the help of Theorem 1, we somewhat extend it onto the anisotropic case.

2.1. Formulations of the basic problems for the anisotropic $p$-Laplacian equation. Let $\mathcal{O} \subset \mathbb{R}_{\boldsymbol{x}}^{N}$ be a polycylinder that meets Condition C. Let $\boldsymbol{p}=\left(p_{1}, p_{2}, \ldots, p_{k}\right), p_{i} \in(1,+\infty)(i=$ $1,2, \ldots, k), p_{*}=\min _{i=1, \ldots, k} p_{i}$. Let $f=f(\boldsymbol{x})$ be given such that

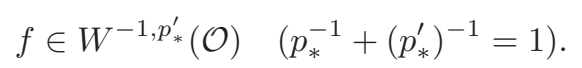

We consider the anisotropic $\boldsymbol{p}$-Laplacian equation $-\sum_{i=1}^{k} \operatorname{div}_{\boldsymbol{x}_{i}}\left(\left|\nabla_{\boldsymbol{x}_{i}} u\right|^{p_{i}-2} \nabla_{\boldsymbol{x}_{i}} u\right)+|u|^{p_{*}^{\prime}-2} u=f$ in $\mathcal{O}$

supplemented with either the Dirichlet boundary condition

$$
u=g \text { on } \partial \mathcal{O},
$$

where $g=g(\boldsymbol{x})$ is given, or the Neumann boundary condition

$$
\sum_{i=1}^{k}\left(\left|\nabla_{\boldsymbol{x}_{i}} u\right|^{p_{i}-2} \nabla_{\boldsymbol{x}_{i}} u\right) \cdot \boldsymbol{\nu}_{i}=h \text { on } \partial \mathcal{O}
$$

where $h=h(\boldsymbol{x})$ is given, or the Robin boundary condition

$\sum_{i=1}^{k}\left(\left|\nabla_{\boldsymbol{x}_{i}} u\right|^{p_{i}-2} \nabla_{\boldsymbol{x}_{i}} u\right) \cdot \boldsymbol{\nu}_{i}+\varkappa|u|^{q_{*}-2} u=\mathfrak{H}$ on $\partial \mathcal{O}$,

where $\frac{1}{p_{*}}-\frac{1-1 / p_{*}}{N-1} \leq \frac{1}{q_{*}}, \mathfrak{H}=\mathfrak{H}(\boldsymbol{x})$ and $\varkappa=$ const $>0$ are given.

In (9), (10), and further, $\boldsymbol{\nu}_{i}$ is the unit outward normal to $\partial \mathcal{B}_{i}$. (If $\boldsymbol{x} \in \partial \mathcal{O}$ but $\boldsymbol{x} \notin \partial \mathcal{B}_{i}$ then in (9) and (10) we simply take zero vector for $\boldsymbol{\nu}_{i}(\boldsymbol{x})$.)

In the isotropic case, the following existence and uniqueness results were established in [11, Chapter 2, Sections 1.6 and 2.3].

Proposition 2. Let $\left(p_{*}=\right) p_{i}=r \in(1,+\infty)$ $(i=1,2, \ldots, k)$. Let $f$ satisfy (6). The following assertions hold true

(i) Whenever $g \in W^{1 / r^{\prime}, r}(\partial \mathcal{O})$, the Dirichlet problem $(7),(8)$ has the unique weak generalized solution $u \in W^{1, r}(\mathcal{O})$.

(ii) Whenever $h \in W^{-1 / r^{\prime}, r^{\prime}}(\partial \mathcal{O})$, the Neumann problem (7), (9) has the unique weak generalized solution $u \in W^{1, r}(\mathcal{O})$.

(iii) Whenever $\mathfrak{H} \in W^{-1 / r^{\prime}, r^{\prime}}(\partial \mathcal{O})$, the Robin problem (7), (10) has the unique weak generalized solution $u \in W^{1, r}(\mathcal{O})$. 
Remark 5. The straightforward application of the monotonicity method, as in [11, Chapter 2], of the Lions - Magenes Trace Theorem, and of the fact that $W^{1, \alpha}(\mathcal{O}) \hookrightarrow W^{1, \beta}(\mathcal{O})$ for $\alpha>\beta$, in the general anisotropic case leads to the assertions of Proposition 2 with $g \in W^{1 / p_{*}^{\prime}, p_{*}}(\partial \mathcal{O}), h \in W^{-1 / p_{*}^{\prime}, p_{*}^{\prime}}(\partial \mathcal{O})$, $\mathfrak{H} \in W^{-1 / p_{*}^{\prime}, p_{*}^{\prime}}(\partial \mathcal{O})$, and $u \in W^{1, p_{*}}(\mathcal{O})$ on the places of $g \in W^{1 / r^{\prime}, r}(\partial \mathcal{O}), h \in W^{-1 / r^{\prime}, r^{\prime}}(\partial \mathcal{O})$, $\mathfrak{H} \in W^{-1 / r^{\prime}, r^{\prime}}(\partial \mathcal{O})$, and $u \in W^{1, r}(\mathcal{O})$. (Here $p_{*}=\min _{i=1, \ldots, k} p_{i}$.) Clearly, the anisotropic peculiarity is ignored in this approach.

2.2. Refinement of regularity via Theorem 1. Applying Theorem 1 we arrive at a more precise conclusion than in Remark 5. This conclusion is the second main result of the article.

Theorem 2. Let $\boldsymbol{p}=\left(p_{1}, p_{2}, \ldots, p_{k}\right), p_{i} \in(1,+\infty)$ $(i=1,2, \ldots, k), p_{*}=\min _{i=1, \ldots, k} p_{i}$. Let $\gamma$ be defined by (4) and $f$ satisfy (6). The following assertions hold true

(i) Whenever $g \in W^{\boldsymbol{\gamma}, \boldsymbol{p}}(\partial \mathcal{O})$, the Dirichlet problem (7), (8) has the unique weak generalized solution $u \in W^{1, p}(\mathcal{O})$.

(ii) Whenever $h \in W^{-\boldsymbol{\gamma}, \boldsymbol{p}^{\prime}}(\partial \mathcal{O})$, the Neumann problem (7), (9) has the unique weak generalized solution $u \in W^{1, \boldsymbol{p}}(\mathcal{O})$.

(iii) Whenever $\mathfrak{H} \in W^{-\boldsymbol{\gamma}, \boldsymbol{p}^{\prime}}(\partial \mathcal{O})$, the Robin problem (7), (10) has the unique weak generalized solution $u \in W^{1, \boldsymbol{p}}(\mathcal{O})$.

Remark 6. Note that $W^{1, \boldsymbol{p}}(\mathcal{O}) \hookrightarrow W^{1, p_{*}}(\mathcal{O})$. Hence Theorem 2 extends the result in Remark 5 indeed.
Remark 7. The approach mentioned above can be also applied to the $\boldsymbol{p}$-parabolic problems. For example, in $\mathcal{O} \times(0, T), T=$ const $>0$ consider the non-stationary equation

$$
u_{t}-\sum_{i=1}^{k} \operatorname{div}_{\boldsymbol{x}_{i}}\left(\left|\nabla_{\boldsymbol{x}_{i}} u\right|^{p_{i}-2} \nabla_{\boldsymbol{x}_{i}} u\right)+|u|^{p_{*}^{\prime}-2} u=f
$$

supplemented with the Cauchy data

$$
\left.u\right|_{t=0}=u_{0} \text { on } \mathcal{O},
$$

and either the Dirichlet condition (8), or the Neumann condition (9), or the Robin condition (10). Let the given functions in (11), (12), (8)-(10) depend, in general, on $t$ (except for $u_{0}$ ) and have the following regularity:

$$
\begin{aligned}
& f \in L^{p_{*}^{\prime}}\left(0, T ; W^{-1, p_{*}^{\prime}}(\mathcal{O})\right), u_{0} \in L^{2}(\mathcal{O}), \\
& g \in L^{p_{*}^{\prime}}\left(0, T ; W^{\boldsymbol{\gamma}, \boldsymbol{p}}(\partial \mathcal{O})\right), \\
& h \in L^{p_{*}^{\prime}}\left(0, T ; W^{-\boldsymbol{\gamma}, \boldsymbol{p}^{\prime}}(\partial \mathcal{O})\right), \\
& \mathfrak{H} \in L^{p_{*}^{\prime}}\left(0, T ; W^{-\gamma, \boldsymbol{p}^{\prime}}(\partial \mathcal{O})\right)+L^{q_{*}^{\prime}}(\mathcal{O} \times\{t=0\}) .
\end{aligned}
$$

Then each of the problems

- (11), (12), (8) (Cauchy - Dirichlet),

- (11), (12), (9) (Cauchy - Neumann),

- (11), (12), (10) (Cauchy - Robin)

has the unique solution $u \in L^{\infty}\left(0, T ; L^{2}(\mathcal{O})\right)$, $\nabla_{\boldsymbol{x}_{i}} u \in L^{p_{i}}(\mathcal{O} \times(0, T))$.

Acknowledgement. The authors are grateful to Dr. Ivan Kuznetsov (Novosibirsk State University) for many fruitful discussions.

\section{Библиографический список}

1. Antontsev S.N., Diaz J.I., and Shmarev S. Energy Methods for Free Boundary Problems. Applications to Nonlinear PDEs and Fluid Mechanics. - Boston, 2002

2. DiBenedetto E. Degenerate Parabolic Equations. - New York, 1993.

3. Vázquez J.L. Smoothing and Decay Estimates for Nonlinear Diffusion Equations. Equations of Porous Medium Type. - New York, 2006.

4. Antontsev S.N. and Kuznetsov I.V. Singular perturbations of forward-backward p-parabolic equations. // JEPE. - 2016. - Vol. 2.

5. Antontsev S.N. and Kuznetsov I.V. Existence of entropy measure-valued solutions for forwardbackward p-parabolic equations. // Sib. Elect. Math. Rep. - 2017. - Vol. 14.

6. Kuznetsov I.V. and Sazhenkov S.A. Anisotropic vanishing diffusion method applied to genuinely nonlinear forward-backward ultraparabolic equations. // Sib. Elect. Math. Rep. 2018. - Vol. 15.

7. Lions J.-L. and Magenes E. Problemi ai limiti non omogenei (III) // Annali della Scuola Normale Superiore di Pisa, Classe di Scienze $3^{e}$ série. 1961. - V. 15, № 1-2. (In Italian.)

8. Lions J.-L. and Magenes E. Problémes aux limites non homogénes et applications, vol. 1 et 2. Paris, 1968. (In French.)

9. Slobodetskii L.N. Generalized S.L. Sobolev spaces and their application to boundary value problems for partial differential equations. Scientific Notes of Leningrad State Ped. Inst. 1958. - Issue 197. (In Russian.)

10. Steinbach O. Numerical Approximation Methods for Elliptic Boundary Value Problems, Finite and Boundary Elements. - New York, 2003. 
11. Lions J.-L. Quelques Méthodes de Résolution des Problémes aux Limites Non Linéaire. - Paris, 1969. (In French.)

12. Kruzhkov S. and Korolev A. Towards a theory of embedding of anisotropic functional spaces. // Dokl. Acad. Nauk. SSSR. - 1985. - V. 285. (In Russian.)
13. Ohno M., Shizuta Y., and Yanagisawa T. The trace theorem on anisotropic Sobolev spaces // Tóhoku Math. J. - 1994. - V. 46.

14. Meyries M. and Schnaubelt R. Interpolation, embeddings and traces of anisotropic fractional Sobolev spaces with temporal weights. // Journal of Functional Analysis. - 2012. - V. 262. 